I NVESTI GACI ÓN

\title{
Relación entre la dimensión socioeconómica y la dimensión salud en familias cubanas
}

\author{
Relationship between the socio-economic dimension \\ and the health dimension in Cuban families
}

Dra. C. Georgia Díaz-Perera Fernández, Dr. C. J orge Bacallao Gallestey, Dr. C. Eduardo Alemañy Pérez

Universidad de Ciencias Médicas de La Habana, Centro de Investigaciones y Referencia de Aterosclerosis de La Habana (CIRAH). La Habana, Cuba.

\section{RESUMEN}

Objetivos: evaluar la magnitud de la relación entre la dimensión socioeconómica y la dimensión salud en familias pertenecientes a diferentes consultorios del médico y la enfermera de la familia.

Métodos: se realizó un estudio transversal en 840 familias pertenecientes a 12 consultorios pertenecientes a siete áreas de salud en La Habana. $L$ a dimensión socioeconómica estuvo representada por condiciones de la vivienda y de la familia, y la de salud por las densidades de hipertensión, diabetes y hábito de fumar. Resultados: no existió asociación entre el nivel de escolaridad y la densidad de diabéticos y fumadores, solo en relación con la hipertensión se apreció algún indicio de asociación lineal con la escolaridad, no hubo indicios de asociación entre las variables de salud y la posesión de equipos de primera y segunda necesidad en la vivienda. Los tres factores de riesgo exhibieron claras tendencias en relación con la percepción de la situación económica. Entre los que aprecian que su condición económica es mala o muy mala, hay densidades altas de las tres entidades, las desigualdades socioeconómicas no se asociaron con la densidad de los factores de riesgo.

Conclusiones: la relación entre las desigualdades socioeconómicas y la situación de salud en Cuba es baja.

Palabras clave: dimensión socioeconómica, salud, hipertensión, diabetes, hábito de fumar, desigualdad, Cuba. 


\section{ABSTRACT}

Objectives: to evaluate the relationship of the socioeconomic dimension and the health dimension in Cuban families cared for by several family physician's offices. Methods: a cross-sectional study of 840 families from 12 family physician's offices located in seven health areas of Havana was conducted. The socioeconomic dimension was represented by the conditions of the house and of the family, and the health dimension was represented by the hypertension, diabetes and smoking densities.

Results: there was no relation between schooling and the density of diabetics and smokers; just hypertension was observed to have some linear relation with schooling. No signs of association between the variables health and the ownership of first and secondary need appliances at home. The three risk factors exhibited clear tendencies in terms of the perception of economic situation. There exist high densities of the three factors among those who considered their economic conditions as bad or very bad; the socio-economic inequalities had no relation with the density of risk factors.

Conclusions: there is low association between socio-economic inequalities and health situation in Cuba.

Key words: socio-economic dimension, health, hypertension, diabetes, smoking, inequality, Cuba.

\section{NTRODUCCI ÓN}

Las condiciones de salud constituyen uno de los indicadores más elocuentes de los logros sociales de una comunidad o de los principios de justicia social en que se funda. La manera de vivir, enfermar y morir de los seres humanos, es la resultante de un variado conjunto de determinantes entre los que desempeñan un importante papel las desigualdades sociales.

Al iniciar el siglo XXI un enorme número de personas sigue malviviendo enferma, desnutrida o subalimentada. La mayoría de los ciudadanos que habitan el planeta no posee el mínimo bienestar material y social que permita un desarrollo adecuado de su salud y que puedan disfrutar y compartir una vida personal y social activa y alegre. En muchos países el estado de salud está empeorando, en otros solo mejora para una parte de la población y, en cualquier caso, la brecha entre los distintos grupos sociales aumenta. ${ }^{1,2}$

El logro de la equidad en salud significa que todas las personas deben tener la misma oportunidad de lograr el pleno desarrollo de su salud, y que nadie debe ser discriminado en su intención de alcanzar ese objetivo. En relación con la salud, podemos definir las inequidades, como aquellas diferencias en salud que son innecesarias, injustas y evitables. ${ }^{2}$

El análisis científico de la desigualdad social en salud contribuye a mostrar dónde, cuándo, cómo, en quién y por qué ocurren diferencias en salud que son evitables e injustas. El conocimiento científico puede ayudar a revelar las desigualdades en salud pero solo la acción social puede reducirlas. Las soluciones técnicas requieren de la voluntad y de la acción política para llevarse a cabo. 
Este trabajo se propone evaluar la magnitud de la relación entre la dimensión socioeconómica y la dimensión salud en familias pertenecientes a 12 consultorios del médico y la enfermera de la familia (CMF) pertenecientes a siete áreas de salud de La Habana.

\section{MÉTODOS}

Se realizó un estudio transversal en 840 familias pertenecientes a $12 \mathrm{CMF}$, seleccionadas a través de un muestreo probabilistico. La dimensión socioeconómica (variable independiente) está representada por condiciones de la vivienda y de la familia, y la de salud por las densidades de hipertensión, diabetes y hábito de fumar. La densidad se define como el cociente entre el total de casos y el total de susceptibles en la familia o vivienda.

Se calcularon los valores promedio y las deviaciones estándar para las tres densidades según estratos de la escolaridad (la más alta entre los miembros del núcleo familiar), los equipos de primera necesidad en la vivienda (radio, plancha, ventilador, televisor, refrigerador y olla de presión), de segunda necesidad (grabadora, batidora, aire acondicionado, tocadiscos, horno microonda, video, máquina de coser, congelador, calentador de agua, otros), y la percepción de la situación económica definida según la percepción de cada individuo encuestado como: 1. Excelente, 2. Buena, 3. Regular, 4. Mala y 5. Muy Mala. Se cuantificó la desigualdad entre viviendas para cada consultorio mediante la varianza de cada una de las variables ya mencionadas y finalmente se calcularon las correlaciones entre las densidades y las medidas de desigualdad.

\section{RESULTADOS}

En la tabla 1 se observa que no existe asociación entre el nivel de escolaridad y la densidad de diabéticos y fumadores, solo en relación con la hipertensión se apreció algún indicio de asociación lineal con la escolaridad.

Tabla 1. Estadisticas descriptivas de las variables de salud de acuerdo a estratos de la escolaridad máxima en el hogar

\begin{tabular}{|c|c|c|c|c|}
\hline \multicolumn{2}{|l|}{ Escolaridad } & $\begin{array}{c}\text { Densidad } \\
\text { de diabéticos } \\
(\mathrm{M} \pm \mathrm{DE})^{*}\end{array}$ & $\begin{array}{c}\text { Densidad } \\
\text { de hipertensos } \\
(M \pm D E)\end{array}$ & $\begin{array}{c}\text { Densidad } \\
\text { de fumadores } \\
(M \pm D E)\end{array}$ \\
\hline \multirow{2}{*}{ Primaria } & Media & $0,09 \pm 0,19$ & $0,29 \pm 0,34$ & $0,19 \pm 0,30$ \\
\hline & $\mathrm{n}$ & 168 & 168 & 168 \\
\hline \multirow{2}{*}{ Secundaria } & Media & $0,08+0,18$ & $0,28+0,30$ & $0,26+0,31$ \\
\hline & $\mathrm{n}$ & 150 & 150 & 150 \\
\hline \multirow{2}{*}{ Preuniversitaria o más } & Media & $0,08+0,16$ & $0,25+0,26$ & $0,22+0,27$ \\
\hline & $\mathrm{n}$ & 522 & 522 & 522 \\
\hline
\end{tabular}

*media \pm desviación estándar.

Fuente: encuesta. 
La tabla 2 muestra que no hay indicios de asociación entre las variables de salud y la posesión de equipos de primera necesidad en la vivienda. Son pocos, comparativamente, los casos que tienen cuatro o menos equipos de primera necesidad, y entre estos núcleos las densidades de diabetes, hipertensión y hábito de fumar no fueron más altas. De manera general los hogares cubanos cuentan con la mayoría de los equipos de primera necesidad.

Tabla 2. Estadisticas descriptivas de las variables de salud de acuerdo a estratos de los equipos de primera necesidad en la vivienda

\begin{tabular}{|c|c|c|c|c|}
\hline \multicolumn{2}{|c|}{ Equipos de primera necesidad } & $\begin{array}{c}\text { Densidad } \\
\text { de diabéticos } \\
(\mathrm{M} \pm \mathrm{DE})^{*}\end{array}$ & $\begin{array}{c}\text { Densidad } \\
\text { de hipertensos } \\
(\mathrm{M} \pm \mathrm{DE})\end{array}$ & $\begin{array}{c}\text { Densidad } \\
\text { de fumadores } \\
\text { (M } \pm \mathrm{DE} \text { ) }\end{array}$ \\
\hline \multirow{2}{*}{40 menos } & Media & $0,08+0,20$ & $0,25+0,29$ & $0,24+0,31$ \\
\cline { 3 - 5 } & Total & 49 & 49 & 49 \\
\hline \multirow{2}{*}{5} & Media & $0,09+0,16$ & $0,29+0,31$ & $0,24+0,31$ \\
\hline & Total & 153 & 153 & 153 \\
\hline \multirow{2}{*}{6} & Media & $0,08+0,17$ & $0,26+0,28$ & $0,22+0,27$ \\
\hline & Total & 638 & 638 & 638 \\
\hline
\end{tabular}

* media \pm desviación estándar.

Fuente: encuesta.

En la tabla 3 se ve que hay también poca variación de las densidades con respecto a la posesión de equipos de segunda necesidad. Existió un gradiente de densidades para el hábito de fumar, un gradiente de densidades muy discreto para la hipertensión, pero no así para la diabetes. Para las dos primeras, las densidades disminuyeron con las mejores condiciones en el hogar.

En la tabla 4 se observa que los tres factores de riesgo (diabetes, hipertensión y hábito de fumar) exhibieron claras tendencias en relación con la percepción de la situación económica. Se tomó la visión pesimista, es decir, la percepción más desfavorable de la condición económica entre los miembros del núcleo familiar.

Tabla 3. Estadísticas descriptivas de las variables de salud de acuerdo a estratos de los equipos de segunda necesidad en la vivienda

\begin{tabular}{|c|c|c|c|c|}
\hline \multicolumn{2}{|c|}{ Equipos de segunda necesidad } & $\begin{array}{c}\text { Densidad } \\
\text { de diabéticos } \\
(\mathrm{M} \pm \mathrm{DE})^{*}\end{array}$ & $\begin{array}{c}\text { Densidad } \\
\text { de hipertensos } \\
(\mathrm{M} \pm \mathrm{DE})\end{array}$ & $\begin{array}{c}\text { Densidad } \\
\text { de fumadores } \\
(\mathrm{M} \pm \mathrm{DE})\end{array}$ \\
\hline \multirow{2}{*}{0} & Media & $0,08+0,17$ & $0,28+0,31$ & $0,26+0,30$ \\
\hline & Total & 302 & 302 & 302 \\
\hline \multirow{2}{*}{$1-2$} & Media & $0,07+0,16$ & $0,26+0,27$ & $0,21+0,26$ \\
\hline & Total & 351 & 351 & 351 \\
\hline \multirow{2}{*}{$3-5$} & Media & $0,10+0,19$ & $0,27+0,27$ & $0,18+0,30$ \\
\hline & Total & 153 & 153 & 153 \\
\hline
\end{tabular}

* mediat desviación estándar.

Fuente: encuesta. 
Tabla 4. Estadísticas descriptivas de las variables de salud de acuerdo a estratos de la percepción de la situación económica a

\begin{tabular}{|c|c|c|c|c|}
\hline \multicolumn{2}{|c|}{$\begin{array}{l}\text { Percepción de la situación } \\
\text { económica }\end{array}$} & \multirow{2}{*}{$\begin{array}{c}\text { Densidad } \\
\text { de diabéticos } \\
(\mathrm{M} \pm \mathrm{DE})^{*} \\
0,07+0,17\end{array}$} & \multirow{2}{*}{$\begin{array}{c}\begin{array}{c}\text { Densidad } \\
\text { de hipertensos } \\
(\mathrm{M} \pm \mathrm{DE})\end{array} \\
0,23+0,25\end{array}$} & \multirow{2}{*}{$\begin{array}{c}\text { Densidad } \\
\text { de fumadores } \\
(M \pm D E)\end{array}$} \\
\hline Excelente o & Media & & & \\
\hline buena & Total & 251 & 251 & 251 \\
\hline \multirow{2}{*}{ Regular } & Media & $0,08+0,16$ & $0,27+0,28$ & $0,24+0,28$ \\
\hline & Total & 454 & 454 & 454 \\
\hline \multirow{2}{*}{$\begin{array}{l}\text { Mala o muy } \\
\text { mala }\end{array}$} & Media & $0,12+0,23$ & $0,32+0,29$ & $0,30+0,35$ \\
\hline & Total & 89 & 89 & 69 \\
\hline
\end{tabular}

a Se refiere a la visión pesimista de la situación económica, *media \pm desviación estándar.

Fuente: encuesta.

Entre los que apreciaron que su condición económica es mala o muy mala, hubo densidades altas de las tres entidades.

Aunque no fue posible formular juicios comparativos entre variables con rangos de variación diferentes, resultó fácil, sin embargo advertir en la tabla 5 que la posesión de equipos de segunda necesidad es más variable que la de equipos de primera necesidad, lo cual apoyó la percepción intuitiva de que en los hogares de la capital hay pocas variaciones con respecto a los equipos de primera necesidad que están virtualmente al alcance de todos. No ocurrió lo mismo con los equipos de segunda necesidad. Otro tanto podría decirse con respecto a estas dos variables, pero en relación con las diferencias entre consultorios. Las mayores desigualdades internas se presentan en el CMF No. 1 y en el CMF No. 6 con los valores más altos de posesión de equipos de segunda necesidad y el CMF No. 2 y el CMF No. 11 con los valores más bajos.

La tabla 6 muestra que las desigualdades socioeconómicas no se asociaron con la densidad de los factores de riesgo. El signo de las correlaciones fue a veces positivo o a veces negativo, pero siempre lejanos de la significación estadística, con la única notable excepción de la densidad de fumadores, que disminuyó a medida que aumentó la posesión de equipos de segunda necesidad. 
Tabla 5. Varianzas de las variables socioeconómicas de la vivienda según consultorio

\begin{tabular}{|c|c|c|c|c|}
\hline $\begin{array}{l}\text { CMF } \\
\text { (No.) }\end{array}$ & $\begin{array}{c}\text { Máxima escolaridad } \\
\text { en el hogar }\end{array}$ & $\begin{array}{l}\text { Peor situación } \\
\text { económica }\end{array}$ & $\begin{array}{l}\text { Equipos } \\
\text { de primera } \\
\text { necesidad }\end{array}$ & $\begin{array}{l}\text { Equipos } \\
\text { de segunda } \\
\text { necesidad }\end{array}$ \\
\hline 1 & 6,18 & 0,43 & 0,44 & 5,05 \\
\hline 2 & 11,84 & 0,34 & 0,51 & 0,51 \\
\hline 3 & 6,31 & 0,51 & 0,87 & 1,59 \\
\hline 4 & 25,89 & 0,29 & 0,44 & 2,44 \\
\hline 5 & 9,31 & 0,17 & 0,31 & 2,39 \\
\hline 6 & 8,07 & 0,65 & 0,22 & 4,90 \\
\hline 7 & 13,53 & 0,69 & 0,23 & 1,22 \\
\hline 8 & 20,45 & 0,48 & 0,08 & 3,91 \\
\hline 9 & 15,63 & 0,65 & 0,31 & 2,82 \\
\hline 10 & 23,02 & 0,44 & 0,15 & 2,11 \\
\hline 11 & 2,22 & 0,44 & 0,93 & 0,84 \\
\hline 12 & 1,66 & 0,27 & 0,38 & 1,03 \\
\hline
\end{tabular}

Fuente: encuesta.

Tabla 6. Correlación entre variables de salud y desigualdades en las variables socioeconómicas

\begin{tabular}{|l|c|c|c|c|}
\hline & $\begin{array}{c}\text { Máxima } \\
\text { escolaridad } \\
\text { en el hogar }\end{array}$ & $\begin{array}{c}\text { Peor situación } \\
\text { económica }\end{array}$ & $\begin{array}{c}\text { Equipos } \\
\text { de primera } \\
\text { necesidad }\end{array}$ & $\begin{array}{c}\text { Equipos } \\
\text { de segunda } \\
\text { necesidad }\end{array}$ \\
\hline \begin{tabular}{|l|c|c|c|} 
Densidad \\
de diabéticos
\end{tabular} & 0,46 & $-0,16$ & $-0,18$ & 0,27 \\
\hline \multirow{2}{*}{$\begin{array}{l}\text { Densidad } \\
\text { de hipertensos }\end{array}$} & 0,12 & 0,60 & 0,55 & 0,38 \\
\hline & $-0,39$ & $-0,56$ & 0,05 & 0,10 \\
\hline $\begin{array}{l}\text { Densidad } \\
\text { de fumadores }\end{array}$ & 0,20 & 0,05 & 0,86 & 0,74 \\
\hline
\end{tabular}

* Correlación significativa para $p<0,01$.

Fuente: encuesta.

\section{DISCUSIÓN}

Las diferencias sustanciales entre los países es un problema en las desigualdades en materia de salud. Estas diferencias tienen lugar en varios ejes de la estratificación social, como el socioeconómico, el político y el cultural. Una forma de describir la magnitud de las desigualdades es la brecha que existe entre el nivel socioeconómico alto y el bajo. Por ejemplo, en El Salvador, si las madres carecen de educación, sus bebés tienen 100 posibilidades en 1000 de morir en el primer año de vida. En cambio, si las madres tienen al menos educación secundaria, la tasa de mortalidad infantil es cuatro veces menor. ${ }^{3,4}$ 
En este estudio no existió asociación entre el nivel de escolaridad y la densidad de diabéticos y fumadores, solo algún indicio de asociación lineal con la hipertensión. Esto puede deberse a que el sistema de salud borra las barreras discriminatorias mediante el acceso universal. En otras sociedades, la escolaridad es un marcador de pobreza.

Las desigualdades sustanciales en materia de salud se manifiestan tanto en los países ricos como en los más pobres. En la ciudad escocesa de Glasgow, la esperanza de vida de los hombres en una de las zonas más marginadas era de 54 años, mientras que en las zonas de mejor condición económica es de 82 años. ${ }^{3}$

Con solo algunas excepciones, se puede demostrar, sobre la base de la evidencia, que cuanto más baja es la posición socioeconómica de un individuo, peor es su salud. Existe un gradiente social en salud que va desde lo más alto a lo más bajo del espectro socioeconómico. Los efectos del gradiente pueden ser drásticamente evidentes y bastante sutiles. En general, las personas que están por encima de la posición inferior presentan un peor estado de salud que las que están por encima de ella, pero un mejor estado de salud que las que están por debajo. ${ }^{5-7}$

Dorling y otros analizaron 126 países del mundo con datos completos sobre la desigualdad de ingresos y la mortalidad por edad y sexo que se disponía en el año 2002, y exploraron si el efecto aparente de la desigualdad de ingresos en la salud, que ha sido demostrado por las naciones más ricas, se replica en todo el mundo, y si el impacto varía según la edad. Los datos se obtuvieron de la OMS y de las Naciones Unidas para el Desarrollo. Encontraron que entre las edades de 15-24 y 25-35 años, las variaciones en la desigualdad de ingresos parecen estar más estrechamente relacionadas con la mortalidad en todo el mundo y que esta relación es especialmente fuerte para los países más pobres de África. La mortalidad es más alta para un determinado nivel de ingresos totales en los países más desiguales. Al parecer, la desigualdad en los ingresos tiene una influencia en todo el mundo, especialmente para los adultos más jóvenes. La desigualdad parece tener un impacto negativo en la salud universal. ${ }^{8}$

En esta investigación no hubo indicios de asociación entre las variables de salud y la posesión de equipos de primera necesidad. De manera general los hogares cubanos cuentan con la mayoría de los equipos de primera necesidad. Este podría ser un caso de asociación debido a escasa variabilidad en la variable independiente, lo cual no niega una asociación real subyacente. En una comunidad en la que todos fuman, el cáncer parecería ser causado por la genética.

Se encontró además poca variación de las densidades con respecto a la posesión de equipos de segunda necesidad.

Se han realizado estudios que relacionan la desigualdad en salud con la prevalencia de diferentes enfermedades, ${ }^{9-12}$ no obstante, el efecto de los factores sociales sobre la disminución de la prevalencia depende fundamentalmente de las políticas e intervenciones que se realicen sobre los factores sociales. El resultado de tabla 4 es importante porque mostró que hay que tomar en cuenta la propia apreciación de la población como indicador de la condición económica. En esta asociación, puede sin embargo, existir una causación o causalidad reversa, en el sentido de que la percepción puede ser efecto de la condición de salud, y no a la inversa.

Estudios que analizan la desigualdad en salud con la hipertensión arterial y enfermedades cardiovasculares, informan que las personas con bajo nivel socioeconómico tienen niveles más altos de tensión arterial y mayor probabilidad de enfermedades cardiovasculares, en parte porque tienen menor acceso a los 
servicios de salud. La mortalidad es asociada con niveles bajos de educación, de ingreso económico y bajos estándares de vida. ${ }^{13-19}$

Otros estudios plantean que pacientes jóvenes con bajo nivel cultural tienen una alta incidencia de mortalidad cardiovascular, tienen menos conocimiento de las enfermedades crónicas y utilizan menos los servicios preventivos. ${ }^{20-24}$

En un estudio realizado en la población cubana de 15 años y más en el año 2001 en las 14 provincias del país y en el municipio I sla de la Juventud, se analizó la relación de la prevalencia de enfermedad cardiovascular con diferentes variables. Se encuentra que la situación económica percibida mostró diferencias significativas ente los grupos de buena y regular, con respecto a los de mala y muy mala. Aquellos con nivel de educación primaria y situación económica mala y muy mala fueron los que exhibieron las prevalencias más elevadas. ${ }^{25}$

En la presente investigación se ha encontrado que los tres problemas de salud estudiados exhiben claras tendencias en relación con la percepción de la situación económica. Esto no significa que la percepción subjetiva de la condición económica sea un indicador fiable de dicha condición, sino que tiene una asociación más alta con la salud que las métricas objetivas habituales de la condición económica.

Se encontró además una asociación débil entre la dimensión socioeconómica y la dimensión salud, o para expresarlo en términos comparativos, mucho más débil que la que registraron la mayoría de los estudios contenidos en la literatura. ${ }^{9,11,12,14,23,26-31}$ La interpretación más plausible es que debido a los principios de equidad, universalidad y no exclusión en que se basa la organización del sistema de salud en Cuba impiden que esas desigualdades socioeconómicas se manifiesten en el terreno de la salud.

Los niveles de salud de la población en el país derivan del papel decisivo del Estado y de la voluntad política de convertir la salud en una política de Estado y en un derecho humano de todos los ciudadanos. Esta política se basa en la inversión en desarrollo social, el desarrollo permanente del sistema y los servicios de salud, la formación de capital humano y social, y en el desarrollo de redes y la participación social.

El Sistema Nacional de Salud (SNS) cubano se sustenta en los siguientes principios: ${ }^{32}$

- La salud es un derecho de la población.

- La salud de la población es responsabilidad del Estado.

- Los servicios de salud alcanzan a toda la población por igual.

- Las prácticas de salud tendrán una sólida base científica.

- Las acciones de salud tendrán una orientación preventiva.

- La participación social es inherente al manejo y desarrollo de los servicios de salud.

- La solidaridad internacional será práctica de los servicios de salud.

Uno de los logros más importantes ha sido el fortalecimiento de la Atención Primaria de Salud (APS) a través del Programa del Médico y la Enfermera de la Familia, el cual permite llevar la atención de salud al punto más cercano posible al lugar donde viven y trabajan las personas. Al fortalecimiento de la APS también ha 
contribuido la participación de las organizaciones de masas y sociales en las tareas de salud.

A pesar de la difícil situación económica de los últimos años, las consecuencias negativas para la población han sido contrarrestadas mediante la cobertura universal y la accesibilidad de los servicios de salud, los gastos en la salud pública se han incrementado de manera estable, lo que refleja la voluntad política de mantener los logros de Cuba en la esfera de la salud.

Se concluye que los niveles de asociación de las desigualdades socioeconómicas en Cuba con la situación de salud son bajos, el sistema de salud en el país se basa en los principios de equidad, universalidad y no exclusión y las consecuencias de la crisis económica para los niveles de salud de la población cubana han sido contrarrestadas mediante la cobertura universal, la accesibilidad de los servicios de salud a todos los niveles y el fortalecimiento del Programa del Médico y la Enfermera de la Familia en la APS.

\section{REFERENCI AS BI BLI OGRÁFICAS}

1. Benach J, Muntaner C. Aprender a mirar la salud. ¿Cómo la desigualdad social daña nuestra salud? Maracay, Venezuela: Instituto de Altos Estudios en Salud Pública "Dr. Arnoldo Gabaldón"; 2005.

2. Rojas F. El desarrollo de la economía global y su impacto sobre políticas de salud. En: Silva Hernández D, editor. Salud Pública Medicina Social. La Habana: Editorial Ciencias Médicas; 2009. p. 193-206.

3. Organización Mundial de la Salud. Lograr la equidad en salud: desde las causas iniciales a los resultados justos. Comisión sobre Determinantes Sociales de la Salud [Internet]. Ginebra, 2008 [citado 2011/3/23]. Disponible en:

http://www.who.int/social_determinants/en/

4. Regidor E. Los determinantes sociales de la salud, la posición socioeconómica y la inequidad en salud. Rev Panam Salud Pública. 2006;20(4):271-2.

5. Fuj isawa Y, Hamano T, Takegawa S. Social capital and perceived health in Japan: An ecological and multilevel analysis. Social Sci Med. 2009;69:500-5.

6. Inaba Y. Social capital and health in Japan: What has been confirmed and what has not. A commentary on I chida and on Fujisawa. Social SciMed. 2009;69: 506-8.

7. Ichida Y, Kondo K, Hirai H, Hanibuchi T, Yoshikawa G, Murata C. Social capital, income inequality and self-rated health in Chita peninsula, Japan: a multilevel analysis of older people in 25 communities Social SciMed. 2009;69:489-99.

8. Dorling D, Mitchell R, Pearce J. The global impact of income inequality on health by age: an observational study. BMJ . 2007;335:873.

9. Schempf AH, Kaufman JS. On the percent of excess risk explained. J Epidemiol Comm Health. 2011;65: 190. 
10. Wright ER, Mills MS, Wright DE. Indiana's Obesity Epidemic Weighs Heavily on Policymakers. Center for Urban Policy and the Environment. 2006 March. [citado 18 Feb 2011]. Disponible en: http://www.urbancenter.iupui.edu

11. Williams DR, Mohammed SA, Leavell J, Collins C. Race, socioeconomic status, and health: Complexities, ongoing challenges, and research opportunities. Ann NY Acad Sci. 2010; 1186: 69101.

12. López R, Baelum V. Inequidades sociales y diabetes en adolescentes. Rev Méd Chile[Internet]. 2006 [citado 24 Ene 2011]; 134(5). Disponible en:

http://www. scielo.cl/scielo.php?pid=S0034-

$\underline{98872006000500002 \& \text { script }=\text { sci_arttext }}$

13. López P, Pradilla LP, Castillo VR, Lahera V. Patologías socioeconómicas como causa de las diferencias regionales en las prevalencias de síndrome metabólico e hipertensión inducida por el embarazo. Rev Esp Cardiol. 2007;60(2): 168-78.

14. Loucks EB, Abrahamowicz M, Xiao Y, Lynch JW. Associations of education with 30 year life course blood pressure trajectories: Framingham Offspring Study. BMC Public Health. 2011,11:139.

15. Kawachi I, AdlerNE, Dow WH. Money, schooling, and health: Mechanisms and causal evidence. Ann NY Acad Sci. 2010; 1186:56-68.

16. Winkleby M, Cubbin C, Ahn D. Low Individual Socioeconomic Status, Neighborhood Socioeconomic Status, and Adult Mortality. Am Jo Public Health. 2006; 96(12): 2145-53.

17. Galobardes B, Morabia A. Measuring the habitat as an indicator of socioeconomic position: methodology and its association with hypertension. J Epidemiol Comm Health. 2003; 57:248-53.

18. Morenoff JD, House JS, Hansen Ben B, Williams DR, Kaplan GA, HunteHaslyn E. Understanding social disparities in hypertension prevalence, awareness, treatment, and control: The role of neighborhood context. Social SciMed. 2007;65: 1853-66.

19. Lynch J, Davey SG, Harper S, Bainbridge K. Explaining the social gradient in coronary heart disease: comparing relative and absolute risk approaches. J

Epidemiol Comm Health. 2006; 60:436-41.

20. Barclay L, Vega C. Poor Health Literacy in the Elderly Predicts All-Cause and Cardiovascular Mortality CME/CE. Arch Intern Med. 2007; 167:1503-9.

21. Bagod'Uva T, O'Donnell O, van Doorslaer E. Differential health reporting by education level and its impact on the measurement of health inequalities among older Europeans. Internat J Epidemiol. 2008; 37: 1375-83.

22. Dow WH, Rehkopf DH. Socioeconomic gradients in health in international and historical context. Ann NY Acad Sci. 2010; 1186:24-36.

23. Adler NE, Stewart J. Health disparities across the lifespan: Meaning, methods, and mechanisms. Ann NY Acad Sci. 2010;1186:5-23. 
24. Evans Gary W, Pilyoung K. Multiple risk exposure as a potential explanatory mechanism for the socioeconomic statushealth gradient. Ann NY Acad Sci. 2010; 1186: 174-89.

25. Armas NB. Enfermedades del corazón y sus características epidemiológicas en la población cubana de 15 años y más. Rev Cubana Invest Bioméd [Internet]. 2009 [citado 24 Feb 2011];28(4). Disponible en:

http://scielo.sld.cu/scielo.php?script=sci_arttext\&pid=S0864$03002009000400004 \&$ lng $=$ es\&nrm=iso\&t|ng=es

26. Matthews KA, Gallo LC, Taylor SE. Are psychosocial factors mediators of socioeconomic status and health connections? A progress report and blueprint for the future. Ann NY Acad Sci. 2010; 1186: 146-73.

27. Pollitt RA, Kaufman JS, Rose KM, Diez-Roux AV, Zeng D, Heiss G. Cumulative life course and adult socioeconomic status and markers of inflammation in adulthood. J of Epidemiol Comm Health. 2008;62:484-91.

28. Martinez A. Social class, marginality and self-assessed health: a cross-sectional analysis of the health gradient in Mexico. Internat J Equity Health. 2009;8:3.

29. Hallqvist J, Lynch J, Bartley M, Lang T, Blane D. Can we disentangle life course processes of accumulation, critical period and social mobility? An analysis of disadvantaged socio-economic positions and myocardial infarction in the Stockholm Heart Epidemiology Program. Social Sci Med. 2004;58: 1555-62.

30. Ebrahim S, Montaner D, Lawlor DA. Clustering of risk factors and social class in childhood and adulthood in British women's heart and health study: cross sectional analysis. BMJ. 2004; 328:861.

31. Brunner E, Shipley MJ, Blane D, Smith GD, Marmot MG. When does cardiovascular risk start? Past and present socioeconomic circumstances and risk factors in adulthood. J Epidemiol Comm Health. 1999; 53:757-64.

32. de la Torre E. El desarrollo del Sistema Nacional de Salud en Cuba. En: Parodi R y Vergara H, editoras. Salud para todos sí es posible. La Habana: Sociedad Cubana de Salud Pública. Sección Medicina Social. 2005. p. 35-93.

Recibido: 30 de junio de 2011.

Aprobado: 2 de febrero de 2012.

Georgia Díaz-Perera Fernández. Centro de Investigaciones y Referencia de Aterosclerosis de La Habana (CIRAH). Policlínico "19 de Abril". Calle Tulipán y Panorama. Nuevo Vedado, Plaza. La Habana, Cuba.

Teléf. 881 4911. Correo electrónico: georgia.diazperera@infomed.sld.cu 